\title{
Twin Trap or Hyphenation of a 3D Paul- and a Cassinian Ion Trap
}

\section{Claus Köster}

Bruker-Daltonik GmbH, Fahrenheitstr. 4, 28359, Bremen, Germany

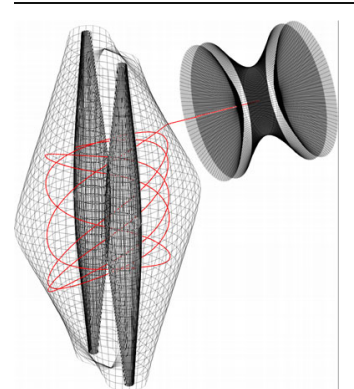

\begin{abstract}
A new, generalized form of electrostatic, harmonic ion trap mass analyzer, referred to as a Cassinian trap, was introduced in 2009. The present work couples a second order Cassinian trap with a 3D Paul trap in an effort to produce an instrument having the advantages of both (i.e., $\mathrm{MS}^{\mathrm{n}}$ and high mass resolution and accuracy). The present study demonstrates the trapping of ions in the 3D Paul trap and their subsequent transfer to the Cassinian trap. The simultaneous transfer of ions over a broad (factor of 13) mass range is shown. Once in the Cassinian trap, ions can be mass analyzed by Fourier transform means, producing resolving powers as high as 53,000 for the fundamental FID and 140,000 for the third harmonic in $1 \mathrm{~s}$.
\end{abstract}

Key words: Cassinian trap, 3D Paul trap, Hyphenation, FT-MS

Received: 26 September 2014/Revised: 8 November 2014/Accepted: 10 November 2014/Published Online: 13 December 2014

\section{Introduction}

A new generalized form of electrostatic harmonic ion trap analyzer (Cassinian trap) was presented in 2009 [1-3]. Such harmonic ion trap mass analyzers are characterized particularly by high mass resolution and high mass accuracy. The more complex form of a Cassinian trap is beneficial for ion injection at stable voltages applied to the trap electrodes. The principle advantages of 3D Paul traps [4] are the ability to accumulate and fragment ions $\left(\mathrm{MS}^{\mathrm{n}}\right)$ [5]. Hyphenating an electrostatic trap with a 3D
Paul trap would ideally provide the capabilities of both analyzers in a single instrument. Such an instrument and corresponding results are described in this article.

\section{Theory}

Equation 1 is a mathematical description of the electric potential, $\Psi$, in a Cassinian trap in a cylindrical coordinate system with the radius (r), the azimuth $(\alpha)$, and the height $(\mathrm{z})$ :

$\Psi(r, \alpha, z)=\left[\frac{\ln \left(\frac{r^{2 n}-2 \cdot \mathrm{r}^{\mathrm{n}} \cdot b^{n} \cdot \cos (\mathrm{n} \alpha)+b^{2 n}}{a^{2 n}}\right)}{\mathrm{Aln}}+\frac{z^{2}-\mathrm{r}^{2} \cdot\left((1-\mathrm{c}) \cdot \cos (\alpha)^{2}+c \cdot \sin (\alpha)^{2}\right)}{\text { Aquad }}+d\right] \cdot \mathrm{U}_{\text {Cassini }}$

$(1)$

where $b$ corresponds to the radius of the trap center to the center of the inner electrodes. If $a$ is smaller than $b$, the inner electrodes will be spatially separated. The individual summands of this equation obey the Laplace equation, and thus represent independent components of

Correspondence to: Claus Köster; e-mail: ck@bdal.de the potential. As a result, further summands, having different constants, can be added to Equation 1, where, Aln (dimensionless) and Aquad (in $\mathrm{m}^{2}$ ) state the portion of the logarithmic-Cassinian and the quadrupolar to the overall potential. The Cassinian voltage, $\mathrm{U}_{\text {Cassini, is the }}$ voltage between the inner and outer electrode; $d$ defines multiplied by $U_{\text {Cassini }}$ a voltage offset; $c$ do not have to be necessarily $1 / 2$, but can be varied to satisfy the Laplace equation. The numerator in the logarithm corresponds to 
the general Cassini Equation 2 [6], hence the name of the trap.

$$
r^{2 n}-2 \cdot \mathrm{r}^{\mathrm{n}} \cdot b^{n} \cdot \cos (\mathrm{n} \alpha)+b^{2 n}=a^{2 n}
$$

Equation 1 describes also the shape of the inner and outer electrodes. Replacing

$\Psi(\mathrm{r}, \alpha, \mathrm{z})$ by the potential of the electrodes and solving the equation for $\mathrm{z}$ yields a function in the other two coordinates. If $\mathrm{n}$ is the Cassinian trap order, then the Kingdon- [7] and the Orbitrap $[8,9]$ are first order Cassinian traps (Figure 1a, Kingdon-trap, first order $\mathrm{n}=1$ and Aquad $=\infty$, Figure 1b, Orbitrap, first order $\mathrm{n}=1$ and $\mathrm{c}=1 / 2$ ). The classic Cassinian trap [in article [1] the classic Cassinian trap was referred as $1^{\text {st }}$ order, but it is more common if the order and the number of inner electrodes are identical], as presented here, is a trap of second order (Figure 1c, $\mathrm{n}=2$ ). A trap with three inner electrodes is of third order (Figure 1d, $n=3$ ).

Possible ion motions were described previously [1]. However, we should differentiate between the harmonic motion in z-direction (i.e., the analytical motion) and the ion motion in the two remaining spatial dimensions (i.e., the trapping motion). The harmonic oscillation along the $\mathrm{z}$-axis is used to analyze the ion's mass because its frequency is proportional to the reciprocal square root of the ion's mass-to-charge ratio $(\mathrm{m} / \mathrm{z})$.

$f_{z}=\frac{1}{2 \cdot \pi} \cdot \frac{\left(2 \cdot \mathrm{e} \cdot \frac{\mathrm{U}_{\text {Cassini }}}{\text { Aquad }}\right)^{1 / 2}}{(m / z)^{1 / 2}}$

The outer electrode is therefore split in half to differentially measure the image current, which is induced by the oscillating ions.

The trapping motion in an Orbitrap is a rotation around the inner electrode. In contrast, one of the possible trapping motions in a classic Cassinian trap is a radial motion between the inner electrodes (i.e., parallel to the y-axis in Figure 2a).

In a classic Cassinian trap, with the potential expressed in Cartesian coordinates,

$\Psi(x, y, z)=\left[\frac{\ln \left(\frac{\left(x^{2}+y^{2}\right)^{2}-2 \cdot b^{2} \cdot\left(x^{2}-y^{2}\right)+b^{4}}{a^{4}}\right)}{\operatorname{Aln}}+\frac{2 \cdot z^{2}-(2-c) \cdot x^{2}-c \cdot y^{2}}{\text { Aquad }}+d\right] \cdot \mathrm{U}_{\text {Cassini }}$

the energy (potential and kinetic) associated with an ion's harmonic motion (i.e., along the z-axis) is preserved and is not available for the motions (i.e., the trapping motion) in the other two directions. For ion stability considerations, only the motion in $\mathrm{x}$ and $\mathrm{y}$ has to be taken into account.

Ion stability can be explained descriptively: A trapped ion is always attracted along the $y$-axis towards $y=0$, so it oscillates

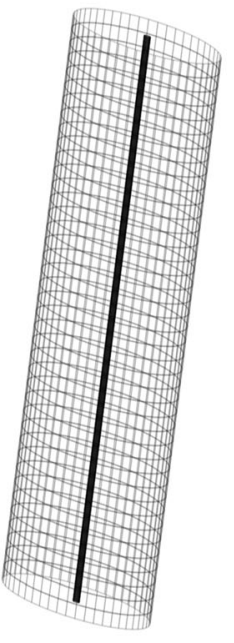

a

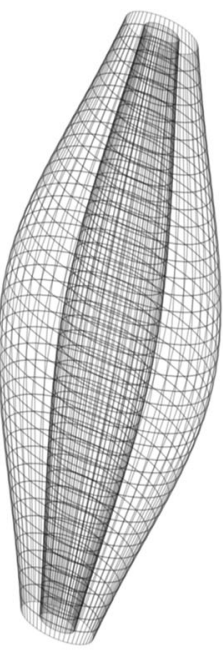

b

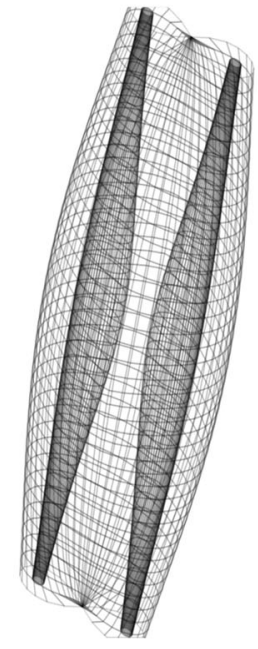

C

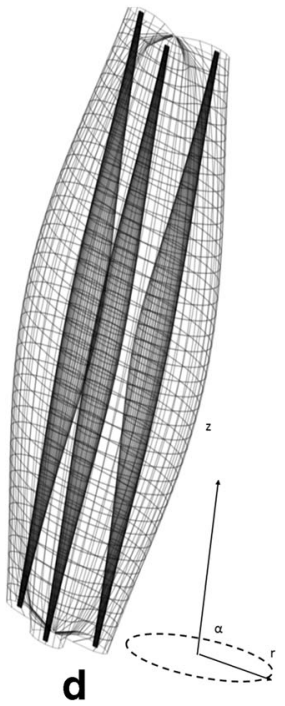

Figure 1. Cassinian traps of different order. (a) Kingdon-trap (first order), (b) Orbitrap (first order), (c) classic Cassinian trap (second order), and (d) third order Cassinian trap 


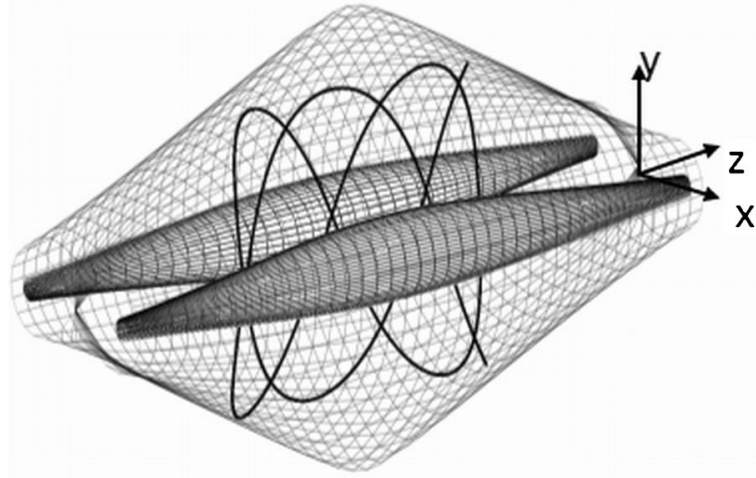

a

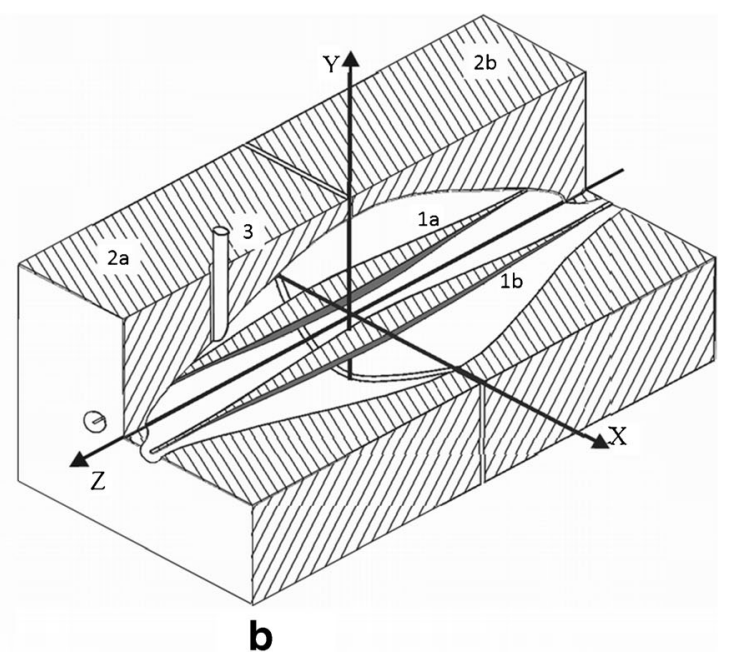

Figure 2. (a) lon motion in a second order Cassinian trap. Harmonic oscillation in the $z$ direction and a radial motion with a large amplitude in y and small amplitude in $\mathbf{x}$. (b) Cross section through a classic Cassinian trap. Inner electrodes (1a and 1b), split outer electrode (2a and $2 b)$, and the trap door (3)

back and forth parallel to the y-axis. At the reversal points, the ion velocity in $\mathrm{y}, \mathrm{v}$ (ystart), is very small so the special probability $1 / v$ (ystart) is very high, hence the ions see for a relatively long time a small, attractive potential in $\mathrm{x}$. The ions see a highly repulsive potential along the $\mathrm{x}$-axis for $\mathrm{y}=0$, but only for a very short time, because the velocity along the $y$-axis is high and so the special probability $1 / \mathrm{v}(\mathrm{y}=0)$ is low. Consequently, the time-averaged potential along the $\mathrm{x}$-axis can be attractive depending on the start position in $\mathrm{y}$. For small $\mathrm{x}$ values, an averaged potential in $\mathrm{x}$ can be defined.

$\Psi_{\text {aver. }}(\mathrm{x}, \mathrm{ystart})=\frac{\int_{y \text { start }}^{-y \text { start }} \frac{\Psi(\mathrm{x}, \mathrm{y}, \mathrm{z})-\Psi(0, \mathrm{y}, \mathrm{z})}{\mathrm{v}(\mathrm{y})} d y}{\int_{\text {ystart }}^{-y \text { start }} \frac{1}{\mathrm{v}(\mathrm{y})} d y}$

The velocity in y can be calculated by:

$\mathrm{v}(\mathrm{y}, \mathrm{ystart})=\left(\frac{2 \cdot \mathrm{e}}{\mathrm{m} / \mathrm{z}} \cdot(\Psi(0, \mathrm{ystart}, \mathrm{z})-\Psi(0, \mathrm{y}, \mathrm{z}))\right)^{1 / 2}$

assuming an initial velocity in y of 0 .

The right starting position in $\mathrm{y}$, ystart, for small $\mathrm{x}$ values, an averaged potential in $\mathrm{x}$ of higher order should be searched. Odd derivatives of $\Psi_{\text {aver. }}$ (x,ystart) with respect to $\mathrm{x}$ are all equal to 0 . So it is necessary to look for ystart values where the second derivative of $\Psi_{\text {aver. }}$ (x,ystart) with respect to $\mathrm{x}$ is 0 . Thus, stable ion trajectories can be expected, when:

$\frac{\mathrm{d}^{2}}{\mathrm{dx}^{2}} \Psi_{\text {aver. }}(0$, ystart $)=0$

The starting position in $\mathrm{z}$ is given when:

$\Psi(0$, ystart, zstart $)=$ Uouter

Uouter should be the potential of the outer electrode.

\section{Methods}

In theory, ions can be started inside the Cassinian trap without having any kinetic energy. One possibility to start ions inside the trap would be to ionize neutrals, e.g., by photo-ionization at the calculated starting conditions $\mathrm{x}=0, \mathrm{y}=\mathrm{ystart}$, and $\mathrm{z}=$ zstart. The ions are trapped and would immediately start their radial and harmonic oscillations.

The injection of ions from outside the trap is more difficult. For positive ion trapping, the outer (detection) electrode would be at ground and the inner electrodes at e.g., $-4 \mathrm{kV}$. When the ions start on some positive potential outside the trap, the ions can penetrate through a hole (drilled in one of the outer electrodes) but would collide with the outer electrode very soon. If a metal tubing (trap door) [10] is set into this hole (no electric contact), a separate voltage (e.g. $-800 \mathrm{~V}$ ) can be applied to it. The trap door is a small metal tubing, typically $1 \mathrm{~mm} \mathrm{o.d} / 0.8$ $\mathrm{mm}$ i.d and $10 \mathrm{~mm}$ long, which is set in the center of a hole (typically $1.5 \mathrm{~mm}$ i.d.) in one side of the outer electrode. The trap door tubing should end with the inner surface of the outer electrode.

Positive ions produced outside the Cassinian trap must start on an electric potential that is lower than the potential of the outer electrode (ground). If the starting potential is e.g., -150 $\mathrm{V}$, the ions can penetrate through the tube very easily and immediately start their travel inside the Cassinian trap. This situation is stable as long as the ions do not come too close to the charged trap door again. In such a case, the ions will be deflected or may escape the trap. However, if the tube potential is pulsed (within $10 \mathrm{~ns}$ ) to ground (or to a voltage that minimizes disturbances caused by the tube) shortly before the ions return to the vicinity of the trap door, they will be trapped for a long time. The time between the initial ion injection (smallest $\mathrm{m} / \mathrm{z}$ of interest) and the closing of the trap door is available for ions with higher $m / z$ ratios to enter the trap. An appropriate setup is shown in Figure $2 b$. The trap door potential needs to be 
switched only for ion injection. The Cassinian voltage can be set to steady voltages, necessary for highly accurate mass measurements.

The "trap door" concept described above makes it possible to couple the Cassinian trap to a wide variety of ion sources or optics. Here, the Cassinian trap is coupled to a 3D Paul trap. Figure 3 depicts the socalled "Twin Trap" of the current work. Elements a to $\mathrm{f}$ include the 3D Paul trap with the detection unit as adopted from a Bruker amaZon instrument. In amaZon mass spectrometers, ions are resonantly ejected out of the 3D Paul trap, accelerated by and focused through lenses $d$ and $\mathrm{e}$, and detected by dynode $\mathrm{f}$ and an electron multiplier (not shown). The 3D Paul trap is comprised of ring electrode $b$ and endcaps a and c.

In the Twin Trap mass spectrometer, electrodes $\mathrm{d}$ to $\mathrm{g}$ are set to steady potentials to guide ions as effectively as possible from the 3D Paul trap into the Cassinian trap. The detection housing and the dynode (Figure 3f) will not be replaced for Twin Trap experiments but set to the same potential. Element $\mathrm{k}$ represents a TOF detector just for use during ion beam alignment. The voltage between the outer and inner Cassini-electrodes remains static.

Ions are accumulated inside the 3D Paul trap by applying a $\mathrm{rf}$ to the ring electrode with a resonant circuit and filling the trap with $\mathrm{He}$ (approximately $2 \cdot 10^{-3} \mathrm{mbar}$ ). The rf has a frequency of $781 \mathrm{kHz}$ and an amplitude of $1200 \mathrm{~V}_{p-p}$. To eject ions out of this trap it is necessary to switch off any rf prior to a high voltage accelerating pulse applied to the endcaps. Because the resonantly applied rf cannot be switched off quickly, a second $\mathrm{rf}$ potential of about $200 \mathrm{kHz}$ and $150 \mathrm{~V}_{p-p}$ is applied nonresonantly to the endcap electrodes. Because it is nonresonant and of relatively low amplitude, this rf can be switched off quickly (i.e., in less than one rf cycle). After ion trapping, the ring electrode amplitude is linearly decreased to $0 \mathrm{~V}$ while the endcap amplitude is

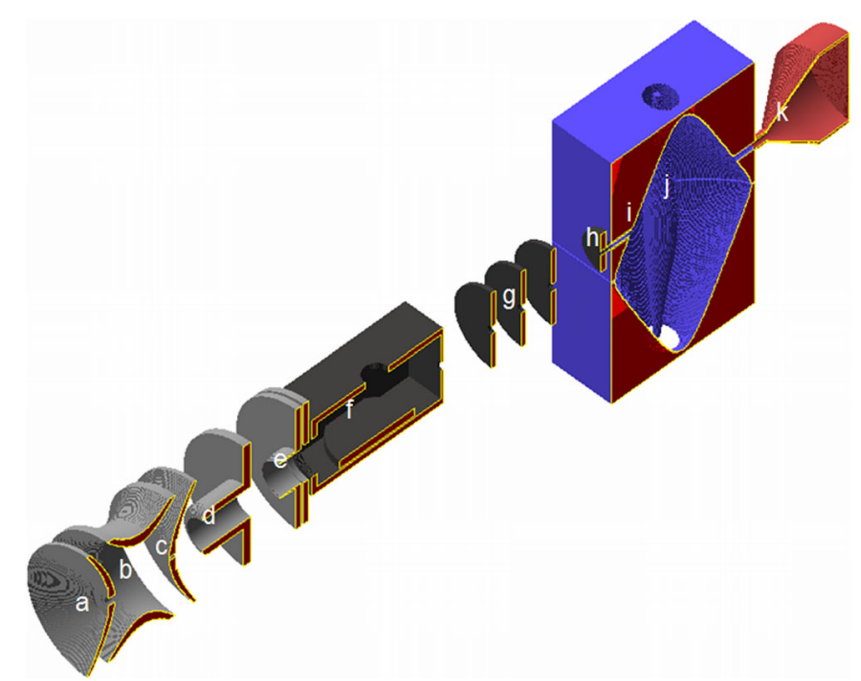

Figure 3. Schematic of a Twin Trap setup (see text) simultaneously increased from $0 \mathrm{~V}$ to $150 \mathrm{~V}_{p-p}$ over a period of $1 \mathrm{~ms}$. The combined $\mathrm{rf}$ on the ring and endcap electrodes holds the ions in the trap throughout this socalled "handshaking" procedure [11]. After a delay of an additional ms, the endcap rf potential is switched off and endcaps a and $\mathrm{c}$ are pulsed to e.g., $300 \mathrm{~V}$ and $-300 \mathrm{~V}$ relative to the trap bias voltage. This ejects the ions out of the 3D Paul trap and accelerates positive ions towards the Cassinian trap.

Before ion ejection, the bias potential of the complete 3D Paul trap (endcaps and ring electrode) is decreased so that the kinetic energy of the ions finally reaching the trap door can be adjusted. After ion ejection the bias is set to ground.

Let us consider a positively charged ion and a voltage of -4 $\mathrm{kV}$ applied to the inner electrode (outer electrode at ground). Ions of a given $\mathrm{m} / \mathrm{z}$ should be injected as a package with appropriate kinetic energy into the Cassini-trap. After ions are accumulated with the 3D Paul trap by applying a $\operatorname{rf}(781 \mathrm{kHz}$, $1200 \mathrm{~V}_{p-p}$ ) to the ring electrode (Figure 3b), the 3D Paul trap bias (endcap and ring electrode) is lowered to $-150 \mathrm{~V}$ (not shown in Figure 4a). Then the rf is switched off by immediately pulsing the entrance end cap to $300 \mathrm{~V}$ (Figure 3a) and the exit end cap (Figure 3c) to $-300 \mathrm{~V}$ relative to the trap bias voltage. This ejects the accumulated ions at a potential of approximately $-150 \mathrm{~V}$. The ions are then accelerated toward the Cassinian trap. The ions enter through the trap, which must be open at e.g., $-800 \mathrm{~V}$. The ions start their motion with large amplitudes in $\mathrm{z}(10 \mathrm{~mm})$ and y (approximately $12 \mathrm{~mm})$ and small amplitude in $\mathrm{x}$ (approximately $\pm 0.5 \mathrm{~mm}$ ). Depending on the ratio of the oscillation period in $\mathrm{y}$ and $\mathrm{z}$, the ions come close to the trap door sooner or later. Then the ions are deflected in $\mathrm{x}$ or escape from the trap. To prevent this, the potential of the trap door must be switched to ground (or e.g., to $-10 \mathrm{~V}$, if the trap door penetrates into the trap a bit more than it should, so as to minimize the disturbance) shortly before the ions return to the vicinity of the trap door. The time between the injection of the first group of ions and their vicinity of the trap door is available for ions with higher $m / z$ to enter the Cassinian trap. The goal is to inject ions with a broad range of $\mathrm{m} / \mathrm{z}$. The mass range can be calculated by:

$\mathrm{c}_{\text {tof }} \cdot\left(m / z_{\text {low }}\right)^{1 / 2}+$ Cycles $\cdot \mathrm{c}_{\text {cassini }} \cdot\left(m / z_{\text {low }}\right)^{1 / 2}=\mathrm{c}_{\text {tof }} \cdot\left(m / z_{\text {high }}\right)^{1 / 2}$

The time-of-flight of the smallest $\mathrm{m} / \mathrm{z}, \mathrm{m} / \mathrm{z}_{\mathrm{low}}$, from the 3D Paul- to the Cassinian trap and inside the Cassinian trap (harmonic cycles before the ion reaches the trap door again) is given on the left side of Equation 9. This time should be equal to the time-of-flight of ions of the highest $m / z, \mathrm{~m} / \mathrm{z}_{\mathrm{high}}$, to reach the Cassinian trap, as given on the right side of Equation 9. Equation 9 rearranges to:

$\frac{\mathrm{m} / \mathrm{z}_{\text {high }}}{\mathrm{m} / \mathrm{z}_{\text {low }}}=\left[1+\text { cycles } \cdot \frac{\mathrm{c}_{\text {cassini }}}{\mathrm{c}_{\text {tof }}}\right]^{2}$

This ratio is high when the time-of-flight region is short and the acceleration voltage is high ( small $_{\mathrm{tof}}$ ). The number of 


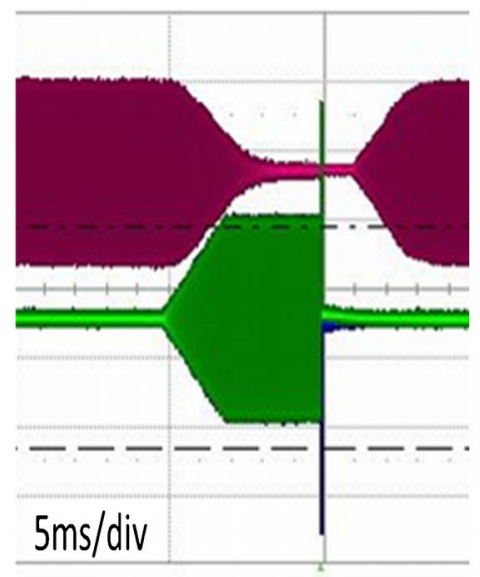

a

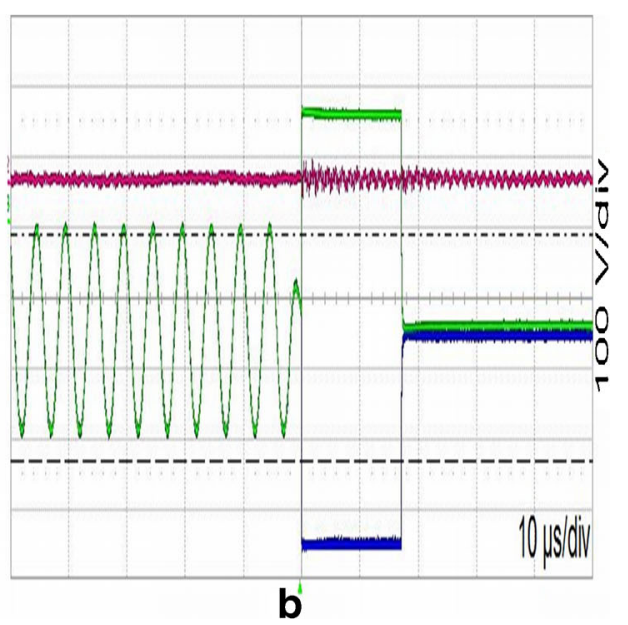

Figure 4. (a) "Handshake." Oscilloscope traces showing the ring electrode if $\left(781 \mathrm{kHz}, 1200 \mathrm{~V}_{p-p}\right)$ in red and endcaps a and c rf (200 $\mathrm{kHz}, 150 \mathrm{~V}_{p-p}$ ) in green and blue, respectively. The high voltage pulses applied to the endcaps for ion ejection can be seen at the end of the rf burst in the blue and greeen traces. The ring electrode if is shown with an offset not to overlay the graphs. (b) A magnification of the HV pulse region in (a)

cycles can be increased by optimizing the Cassinian trap parameters based on the ratio of initial ion motion frequencies in the $z$ - and y-direction. SIMION calculations show that the trap door open voltage has also a big influence on the first ion motion cycles. When the Cassinian voltage is increased by constant trap door voltage, this influence becomes smaller and the number of cycles can be increased.

After ion injection, the image current induced by the ions is differentially measured. A Fourier transformation of the signal (e.g., $1 \mathrm{~s}$ transient) gives a frequency spectrum that results, after calibration, in a mass spectrum.

While the ions are detected inside the Cassinian trap for e.g., $1 \mathrm{~s}$, new ions will be trapped by the 3D Paul trap and the "handshake" procedure can be replaced by normal "amaZon" ion detection, where the ions are ejected out of the 3D Paul trap by resonant ejection [12]. The Bruker amaZon instrument has a maximum scan speed of $52,000 \mathrm{Da} / \mathrm{s}$, so a mass range of 1500 Da can be measured within $29 \mathrm{~ms}$. With ion accumulation and some electronic extra time, 20 overview mass spectra can easily be recorded, while one high resolution mass spectrum is measured.

\section{Experimental}

A prototype Bruker amaZon mass spectrometer serves as the foundation for the hybrid instrument depicted in Figure 3. The 3D Paul trap manifold was extended beyond the detection unit by two differentially pumped manifolds. In the 3D Paul trap manifold (Figure $3 \mathrm{a}-\mathrm{f}$ ), a pressure of about $5 \cdot 10^{-6} \mathrm{mbar}$, in the lens stack manifold (Figure $3 \mathrm{~g}$ ) a pressure of $2 \cdot 10^{-8}$ mbar and in the Cassinian trap manifold (Figure 3i-k) a pressure of $2 \cdot 10^{-10}$ mbar could be maintained. The parameters of the inserted Cassinian trap can be summarized as follows: $\mathrm{n}=2, \mathrm{~b}=7 \mathrm{~mm}, \mathrm{a}=6.5$ $\mathrm{mm}$, Aln $=0.681, \mathrm{c}=1.75$, Aquad $=1017.5 \mathrm{~mm}^{2}, \mathrm{c}=$ -0.042 . The high voltage power switches for pulsing the endcaps were taken from the Bruker OTOF product line and the image current detection from a Bruker FTMS. Accumulation time in the 3D Paul trap was typically 25 $\mathrm{ms}$. The transfer voltages are: $\mathrm{U}_{\mathrm{d}}=-600 \mathrm{~V}, \mathrm{U}_{\mathrm{e}}=\mathrm{U}_{\mathrm{f}}=-$ $8000 \mathrm{~V}, \mathrm{U}_{\text {gleft }}=-6000 \mathrm{~V}, \mathrm{U}_{\text {gcenter }}=-3200 \mathrm{~V}$ and $\mathrm{U}_{\text {gright }}$ $=-1800 \mathrm{~V}$. Analytes were dissolved in $\left(\mathrm{H}_{2} \mathrm{O}: \mathrm{CH}_{3} \mathrm{CN}, 1: 1\right.$ with $0.1 \%$ TFA) to give a concentration of $1 \mathrm{pmol} / \mu \mathrm{L}$ and were infused via the Apollo II electrospray ion source equipped with dual funnel transfer, at the flow rate of $3 \mu \mathrm{L} / \mathrm{min}$.

\section{Results}

Agilent ESI Tuning Mix for ion trap gave a mass spectrum shown in Figure 5. A two point calibration with mass 118.086255 Da (f= 403.61834 kHz) and 922.009798 Da ( $\mathrm{f}=$ $144.4268 \mathrm{kHz}$ ) results in an internal mass accuracy of $0.24 \mathrm{ppm}$ at mass $322.048121 \mathrm{Da}$ and $-0.03 \mathrm{ppm}$ at mass 622.028960 $\mathrm{Da}$. The corresponding Cassinian voltage was calculated to 4004.492 V.

The measured mass range of $\mathrm{m}_{\text {high }} / \mathrm{m}_{\text {low }}=1522 / 118 \approx$ 13 is in good accordance with SIMION calculation and corresponds to three cycles (Equation 10) of the smallest $m / z$ value 118 Da.

In the following are shown $1 \mathrm{~s}$ narrow band FIDs and mass spectra acquired with single zero filling and sine wave apodization. As shown in Figure 6a, the fundamental frequencies of the double protonated LHRH were measured for $1 \mathrm{~s}$ to give a mass resolution of 53,000.

The ion motion in the z-direction is harmonic; however, the corresponding signal is not a pure sine or cosine wave. This 


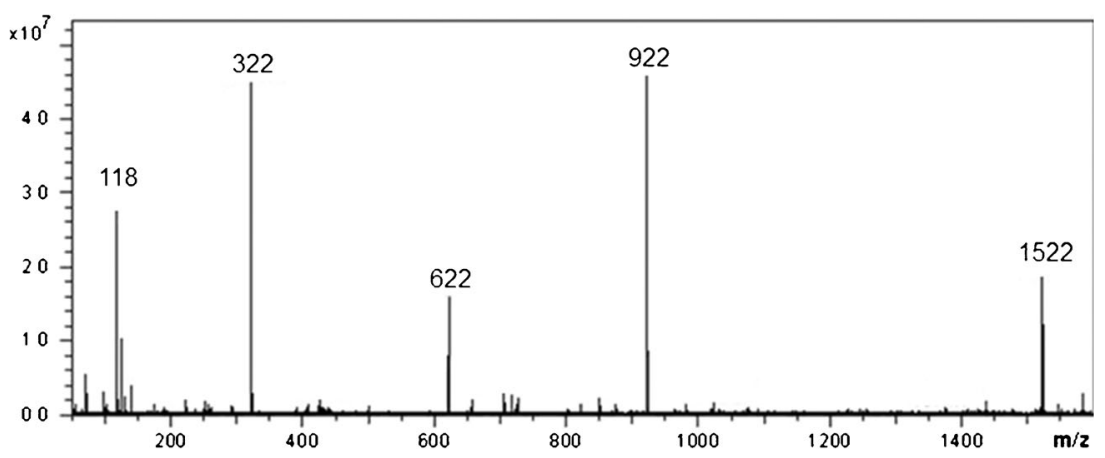

Figure 5. Mass spectrum of Agilent ESI tune mix for ion trap to show a mass range of $\mathrm{m}_{\text {high }} / \mathrm{m}_{\text {low }}=1522 / 118 \approx 13$

results in odd multiples overtones. Even overtones have not been observed. For example, the third harmonic of the above FID with about $8 \%$ of the fundamental height is shown in Figure 6 b. The FID and mass spectrum of bovine insulin ${ }^{6 \mathrm{H}^{+}}$ are shown in Figure 6c.

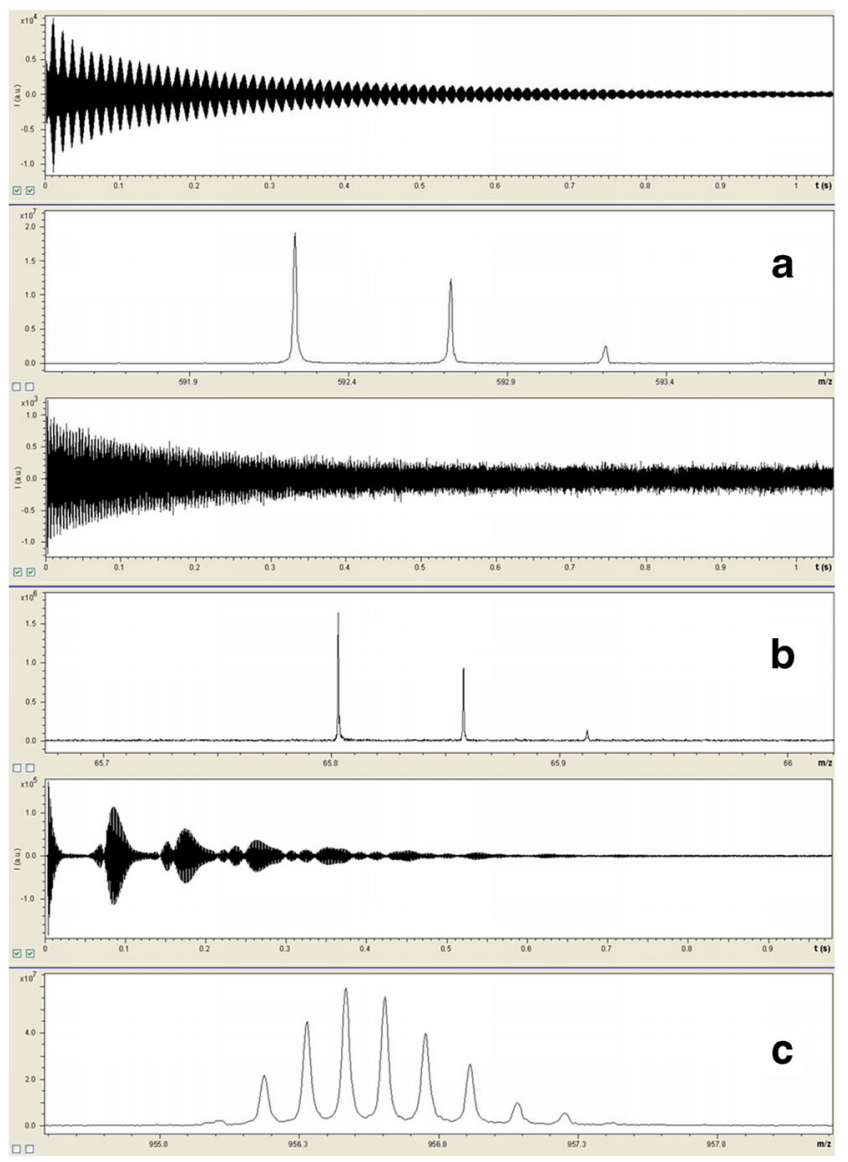

Figure 6. (a) Narrowband $1 \mathrm{~s}$ fundamental FID (upper) and mass spectrum of LHRH (lower) showing a mass resolution of 53,000. (b) Narrowband $1 \mathrm{~s}$ third harmonic FID (upper) and mass spectrum of LHRH (lower) with a mass resolution of 140,000. (c) Narrowband 1 s FID (upper) and mass spectrum (lower) of bovine insulin ${ }^{6 \mathrm{H}+}$ with a mass resolution of 25,000

\section{Conclusion}

The coupling of a 3D Paul trap to a Cassinian ion trap was demonstrated. A so-called "handshake" is an essential process in this coupling. In this process, ions are first accumulated in the 3D Paul trap by a resonantly applied rf potential on the ring electrode. After accumulation, the resonant $\mathrm{rf}$ is turned off and the ions are exclusively trapped by a non-resonant rf potential applied between the end cap electrodes. This non-resonant $\mathrm{rf}$ can be abruptly switched off, after which a HV pulse can be applied to the end caps so that the ions are ejected towards the Cassinian trap. Lowering of the 3D Paul trap bias prior to the HV pulse adjusts the kinetic energy the ions have when entering the Cassinian trap. A "trapdoor" electrode allows ion injection into the Cassinian trap. First results show that the prototype setup gives a mass range, $\mathrm{m}_{\text {high }} / \mathrm{m}_{\text {low }}$, of 13 and an internal mass accuracy of better that $1 \mathrm{ppm}$. A mass resolution of 53,000 was measured at mass $592 \mathrm{Da}\left(\mathrm{LHRH}^{2 \mathrm{H}+}\right)$ at its fundamental frequency, and a resolution of 140,000 was achieved at its third harmonic. Bovine insulin ${ }^{6 \mathrm{H}^{+}}$gave a mass resolution of 25,000 .

A better sensitivity and mass range can be achieved by optimizing ion optics between the 3D Paul- and Cassinian trap. An overall smaller Cassinian trap, higher Cassinian trap voltages, and using the absorption mode instead of the magnitude mode to do the $\mathrm{ftt}$ should increase the mass resolution by a factor of four to five.

Questions regarding the sensitivity, space charge effects, or comparison with other electrostatic traps cannot be addressed because this project was canceled immediately after acquiring the presented results.

\section{Acknowledgment}

The author thanks Dr. Christoph Gebhardt for his help to set up the Twin Trap instrument and for his patience in endless discussions. 


\section{References}

1. Köster, C.: The concept of electrostatic non-orbital harmonic ion trapping. Int. J. Mass Spectrom. 287, 114-118 (2009)

2. Köster, C.: Massenspektrometer mit einer elektrostatischen Ionenfalle. German Patent DE 102007024858 B4 (2007)

3. Köster, C.: Kingdon-Ionenfallen mit Cassini-Potentialen höherer Ordnung. German Patent DE 102011008713 B4 (2011)

4. Paul, W., Steinwedel, H.: Verfahren zur Trennung bzw. zum getrennten Nachweis von Ionen verschiedener spezifischer Ladung. German Patent DE 944900 C (1953)

5. March, R.E., Todd, J.F.J.: Quadrupole ion trap mass spectrometry, 2nd edn, Wiley \& Sons, New York (2005)

6. Available at: www.2dcurves.com. Accessed January 042011
7. Kingdon, K.H.: A method for neutralizing the electron space charge by positive ionization at very low pressure. Phys. Rev. 21, 408-418 (1923)

8. Makarov, A.A.: Electrostatic axially harmonic orbital trapping: a highperformance technique of mass analysis. Anal. Chem. 72, 1156-1162 (2000)

9. Makarov, A.A.: Mass spectrometer. U.S. Patent 5886346 (1999)

10. Köster, C., Franzen, J.: Einspeichern von Ionen in Kíngdon-Ionenfallen. German Patent DE 10200902886 B4 (2009)

11. Brekenfeld, A., Gebhardt, C., Hartmer, R.: Auswerfen einer lonenwolke aus 3D-HF-lonenfallen. German Patent DE 102012013038 A1 (2012)

12. Franzen, J.: Non-linear ion trap. Part 5. Nature of non-linear resonance and resonant ion ejection. Int. J. Mass Spectrom. Ion Process. 130, 15-40 (1994) 